\title{
Adapted Control Methods for Cerebral Palsy Users of an Intelligent Wheelchair
}

\author{
Brígida Mónica Faria ${ }^{1,2,3,4}$, Luis Paulo Reis ${ }^{1,5}$, Nuno Lau ${ }^{2,3}$ \\ ${ }^{1}$ Laboratório de Inteligência Artificial e Ciência de Computadores (LIACC), ${ }^{2}$ Inst. \\ Eng. Electrónica e Telemática de Aveiro (IEETA), ${ }^{3}$ Dep. Electrónica, \\ Telecomunicações e Informática da Universidade de Aveiro (DETI/UA), ${ }^{4}$ Escola \\ Superior Tecnologia de Saúde do Porto / Instituto Politécnico do Porto \\ (ESTSP/IPP), ${ }^{5}$ Dep. Sistemas de Informação, Escola de Engenharia da \\ Universidade do Minho (DSI/EEUM) \\ Emails: btf@estsp.ipp.pt, lpreis@dsi.uminho.pt, nunolau@ua.pt
}

\begin{abstract}
The development of an intelligent wheelchair (IW) platform that may be easily adapted to any commercial electric powered wheelchair and aid any person with special mobility needs is the main objective of the IntelllWheels project. To be able to achieve this main objective, three distinct control methods were implemented in the IW: manual, shared and automatic. Several algorithms were developed for each of these control methods. This paper presents three of the most significant of those algorithms with emphasis on the shared control method. Experiments were performed by users suffering from cerebral palsy, using a realistic simulator, in order to validate the approach. The experiments revealed the importance of using shared (aided) controls for users with severe disabilities. The patients still felt having complete control over the wheelchair movement when using a shared control at a 50\% level and thus this control type was very well accepted. Thus it may be used in intelligent wheelchairs since it is able to correct the direction in case of involuntary movements of the user but still gives him a sense of complete control over the IW movement.
\end{abstract}

Keywords - Intelligent Robotics, Intelligent Systems, Intelligent Wheelchair, Shared Control, Cerebral Palsy

\section{Introduction}

Scientific research allowed the evolution and development of many technologies that are nowadays used in everyday life. In particular, innovations in the field of assistive technologies enabled increased autonomy and independence for human beings that, for some reason, have some kind of disability. Intelligent wheelchairs are an obvious application of the scientific work developed in the last decades on this area. Moreover, these assistive technologies still are object of research and the interaction between them and the user is still an open research problem. The interaction between the Human and the IW is an important component to take in 
consideration. The users' opinions should also be integrated in the development process of the instruments which are to serve and fulfill a human necessity.

The electric wheelchairs are assistive technologies and are typically driven by two individually powered wheels which rotate around a horizontal axis, and another two non-powered caster wheels, which, besides rotating around a horizontal axis, also have the ability to rotate around a vertical axis [1]. IW manual control is, in general, performed through the use of a joystick. The mapping of joystick positions to individual wheel speeds can be performed in many ways and it is this mapping that will determine the manual control behavior. For that reason, several of these mappings were implemented and tested with real users in a simulated environment and based on the users' feedback some interesting conclusions about mappings were achieved [1]. Nevertheless, an IW can also be controlled in a high level manner, for example an action such as "going to the bedroom" can be performed autonomously by the IW. In shared control, the navigation process is divided between patient and machine. In this case, it is the machine which takes control when the navigation of the patient endangers its own safety, in situations such as potential collisions with objects [2] and aids the patient in his IW navigation by recognizing his intentions and filtering his involuntary inputs.

This paper is organized in 5 sections. The first section is composed by this introduction. The second section reports the related work about Intelligent Wheelchairs including a brief description about the IntellWheels project. The implementations of the proposed methods for manual, shared and automatic control are described in the third section. Next, the experimental work and results are presented. Finally some conclusions and directions for future work conclude the paper.

\section{Intelligent Wheelchairs}

In the last years several prototypes of IW have been developed and many scientific works have been published [3-5] in this area. Next, the state of art related to intelligent wheelchairs with special attention to the work developed in the IntellWheels project is presented. 


\subsection{Intelligent Wheelchairs' Developments}

Nowadays, science allows having intelligent wheelchairs very similar in shape to traditional wheelchairs, with high maneuverability and navigational intelligence, with units that can be attached and/or removed and with more power autonomy [5]. A simple definition of Intelligent Wheelchair is a locomotion device used to assist a user having some kind of physical disability, where an artificial control system augments or replaces the user control [6-7]. The main objective is to reduce or eliminate the user's task of having to drive a motorized wheelchair.

Table 1. Intelligent Wheelchairs' projects

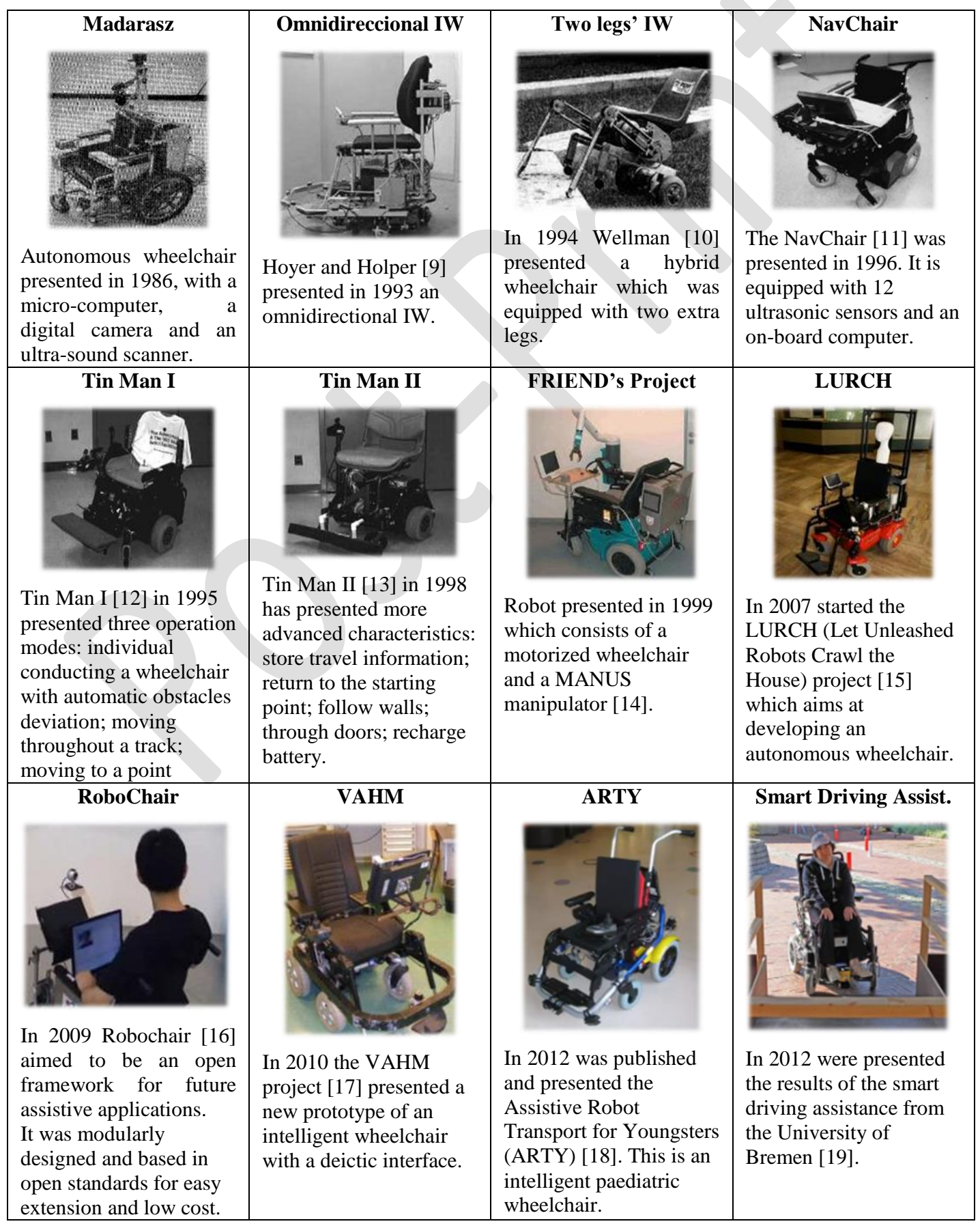


Usually, an IW is controlled by a computer, has a set of sensors and applies techniques derived from mobile robotics research in order to process the sensor information and generate the motors commands in an automatic way or with a shared control. The interface may consist of a conventional wheelchair joystick, voice based control, facial expressions or even gaze control, among others. The developments since 1986 when the Madarasz project [8] was first proposed, evolved all over the world. Several new projects were presented. Table 1 presents a list of some IW prototypes and describes some of their characteristics.

It is possible to observe several solutions with complex ergonomics, however there are also some projects that try to considerer the users' opinions about the modifications to their wheelchairs. The project presented in the next section tries to follow the principle of introducing in a conventional wheelchair a simple platform that can transform it into an intelligent wheelchair.

\subsection{IntellWheels Project}

The IntellWheels project aims to develop an intelligent wheelchair platform that may be easily adapted to any commercial wheelchair and assist any person with special mobility needs [20]. The project main focus is the research and design of a multi-agent platform, enabling easy integration of different sensors, actuators, devices for extended interaction with the user, navigation methods and planning techniques and methodologies for intelligent cooperation to solve problems associated with intelligent wheelchairs [21].

A real prototype (Figure 1) was created by adapting a typical electric wheelchair. Two side bars with a total of 16 sonars, a laser range finder and two encoders were incorporated. 

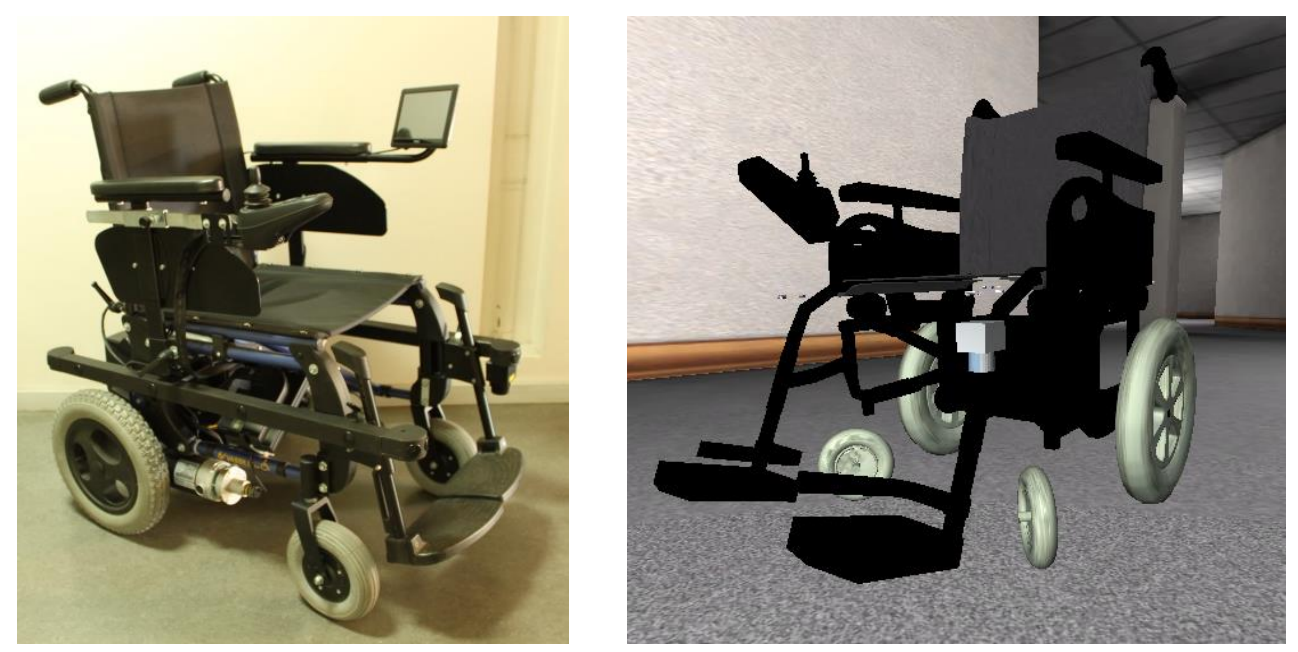

Figure 1. Real prototype of the IW

In the scope of the IntellWheels project, an IW simulator, called IntellSim, was developed. The objective of this simulator is essentially to support the test of algorithms, analyze and test the modules of the platform and safely train users of the IW in a simulated environment [5] [22]. In Figure 1 (right side) it is possible to observe the modeled virtual wheelchair.

A multimodal interface was also developed that allows driving the wheelchair with several inputs such as joystick, gamepad, head movements or more high level commands such as voice commands and facial expressions recognized by a brain computer interface or even with a combination of these input methods. For example it is possible to blink an eye and say "go" for the wheelchair to follow a right wall [23-25]. Therefore it is possible to drive the IW in a completely automatic way, with a shared control or using the usual manual control.

\section{Manual, Automatic and Shared Controls}

One of the main objectives of the IntellWheels project was to be able to control the wheelchair using three distinct methods: manual, shared and automatic [26]. During the course of the project several manual and shared algorithms have been developed, but their comparative evaluation had never been performed. In the course of this work several experiments were performed in order to compare the algorithms developed. These experiments provided the best manual, shared or automatic control adapted to the patients. 


\subsection{System Architecture}

The system is composed by eight main modules and enables a therapist to make experiments with users using distinct types of control methods (Figure 2).

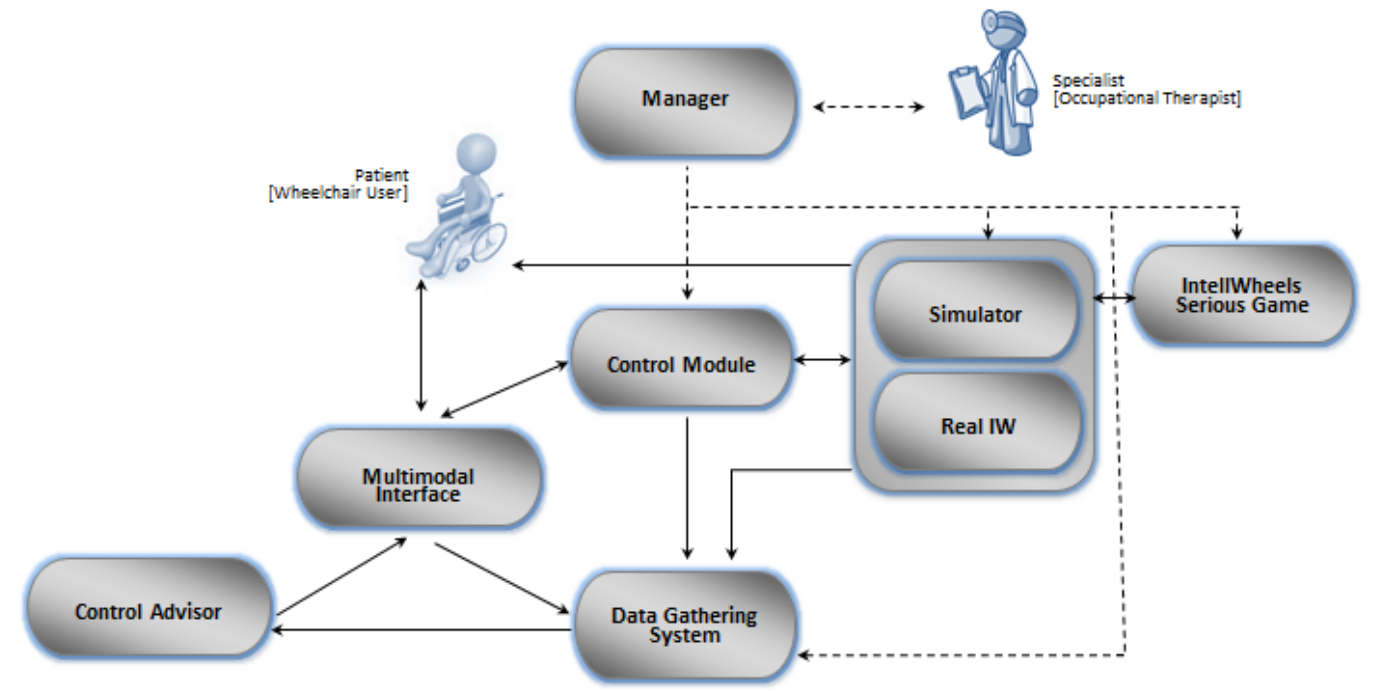

Figure 2. System architecture

The core of the system is composed by the Intelwheels multimodal interface and Intellwheels control module. The multimodal interface enables the patient to fully control real and simulated Intelligent Wheelchairs, using multimodal inputs, including pre-defined input sequences that may be freely associated with any of the available outputs (wheelchair actions).

The interface is connected to the control module that is able to receive high-level or medium level commands from the multimodal interface and control a real or simulated wheelchair making it perform the actions corresponding to those commands (such as go ahead, turn right, follow the right wall, stop, among many others). The control module is also responsible for providing the manual, shared and automatic control modes. The last two modes enable users with disabilities to control the wheelchair using intentions without the need of performing fine-tuned movements/inputs.

The control module may control the real IW prototype but also, in exactly the same way, the simulated intelligent wheelchair in the context of the simulator. This simulator was built using Unreal Tournament 2004 and USARSim 3.1.3 together with a realistic scenario developed with Unreal 2004 Editor. This 3D scenario is a very realistic model of the cerebral palsy institution facilities, thus enabling the development of experiments, in a simulated mode, in a scenario very similar to the one the patients were used to. 
In order to be able to develop and conduct meaningful experiments, a serious game for intelligent wheelchair teaching and testing was built. The game permits the definition of circuits and the placement of markers (balls and stars) that must be collected by the user in order to gain points. It also enables gathering other performance measures such as the trajectory of the users performing the circuit.

In order to be able to extract user profiles and adapt the user interface to the users' profiles several other applications were developed. The first one consists on a complete data gathering system that is able to gather the data available on: multimodal interface; control module; simulated wheelchair; real wheelchair and serious game, and then synchronize all of this data and freely select the values to record in appropriate files in order to be further analyzed by the data analysis applications. A user profiling application integrated in the multimodal interface was also created in this context, in order to be able to conduct controlled experiments with each user and to analyze their capabilities of performing each type of possible input in each of the available input devices.

Based on the user profiling and associated data gathering system, a data analysis system was developed enabling the analysis of users capabilities when performing each type of input and when driving the IW with different input combinations and using distinct control modes (normal and shared with distinct levels of handicap). This module is able to advise, in a simple manner, the best control mode for each user.

A manager module was also developed in order to be able to perform a large set of experiments using the developed user profile extraction methodology and the set of implemented applications. This manager allows to launch all the applications, perform user profiling tests, define the scenario to be used, the circuit to be performed, the control modes to be tested and the data to be gathered and analyzed.

\subsection{Manual Control}

The mapping of joystick positions to individual wheel speeds can be done in an infinite number of combinations, and it is this mapping that will determine the behavior of the wheelchair in response to manual control.

Considering that the joystick handle position is represented in a Cartesian coordinate system, with two axis, $\mathrm{x}$ and $\mathrm{y}$, which vary between -1 and 1 . These (x, 
y) coordinates can be used to determine the distance of the handle to the central (resting) position of the joystick $(0,0)$ and an angle relating to a reference vector (which is usually $(0,1)$ ). The desired speed of the left wheel $(\mathrm{L})$ or the right wheel $(\mathrm{R})$ is represented by normalized values (between -1 and 1 ). With positive values the wheels rotate forward and with negative values the wheels rotate backward. An intuitive mapping was implemented and the equations for $\mathrm{R}$ and $\mathrm{L}$ are:

$$
\left\{\begin{array}{l}
R=y-n x \\
L=y+n x
\end{array}\right.
$$

the value $n x$ follows Equation (2):

$$
n x=\left\{\begin{array}{l}
u_{1} c_{\text {point }}+\left(x-c_{\text {point }}\right) \times u_{2} \quad \text { if } x>c_{\text {point }} \\
-u_{1} c_{\text {point }}+\left(x+c_{\text {point }}\right) \times u_{2} \quad \text { if } x<-c_{\text {point }} \\
u_{1} x \text { if }-c_{\text {point }} \leq x \leq c_{\text {point }}
\end{array}\right.
$$

where $c_{\text {point }} \in[0,1] ; u_{1} \in[0,1]$ and $u_{2} \in[0,1]$. The tested values were $c_{\text {point }}=0.2 ; u_{1}$ $=0.5$ and $u_{2}=0.25$. The first slope $u_{1}$ allows a fast curve and the next slope $u_{2}$ after the cut point $\left(c_{\text {point }}\right)$ should allow a slower curve.

\subsection{Automatic Control}

The developed automatic control has as main objective following a predefined circuit by passing waypoints without the need of user intervention. The automatic control assumes full control over the navigation of the wheelchair and executes the navigating task following the circuit points without any user's intervention. A predefined circuit can be specified by defining the relevant circuit points and the wheelchair can autonomously drive to the specified points.

The main reason to create an automatic control was concerned with the methodology used to achieve a shared driving algorithm for the intelligent wheelchair. The users in the study performed predetermined tasks such as collecting objects along a circuit. This automatic control assumes the selflocalization problem solved and hence the IW always knows its position in the environment. Before this work, the IntellWheels IW was already capable of some forms of automatic control that did not rely on localization, such as "follow wall" or other high level actions like "go forward" [23]. However, it still missed an 
automatic driving algorithm based on the current position and orientation and the desired position and orientation.

Figure 3 shows the idea of the implemented algorithm. If the IW would move directly from target to target, it would have to stop and turn on the spot at each target. This is a very unnatural way of driving the IW. In order to have a smoother path, The IW should consider not only the following target, but also the target that follows. The position of the wheelchair in the world referential $(\mathrm{x}, \mathrm{y})$ combined along with the position of the target (T) and the next target (NT) in order to determine a corrected target (CT) of the trajectory.

$$
C T=T+d_{[C T, T]} \times \frac{\overrightarrow{N T T}}{\|\overrightarrow{N T T}\|}
$$

With the introduction of the corrected target it is possible to have a smoother trajectory of the wheelchair.

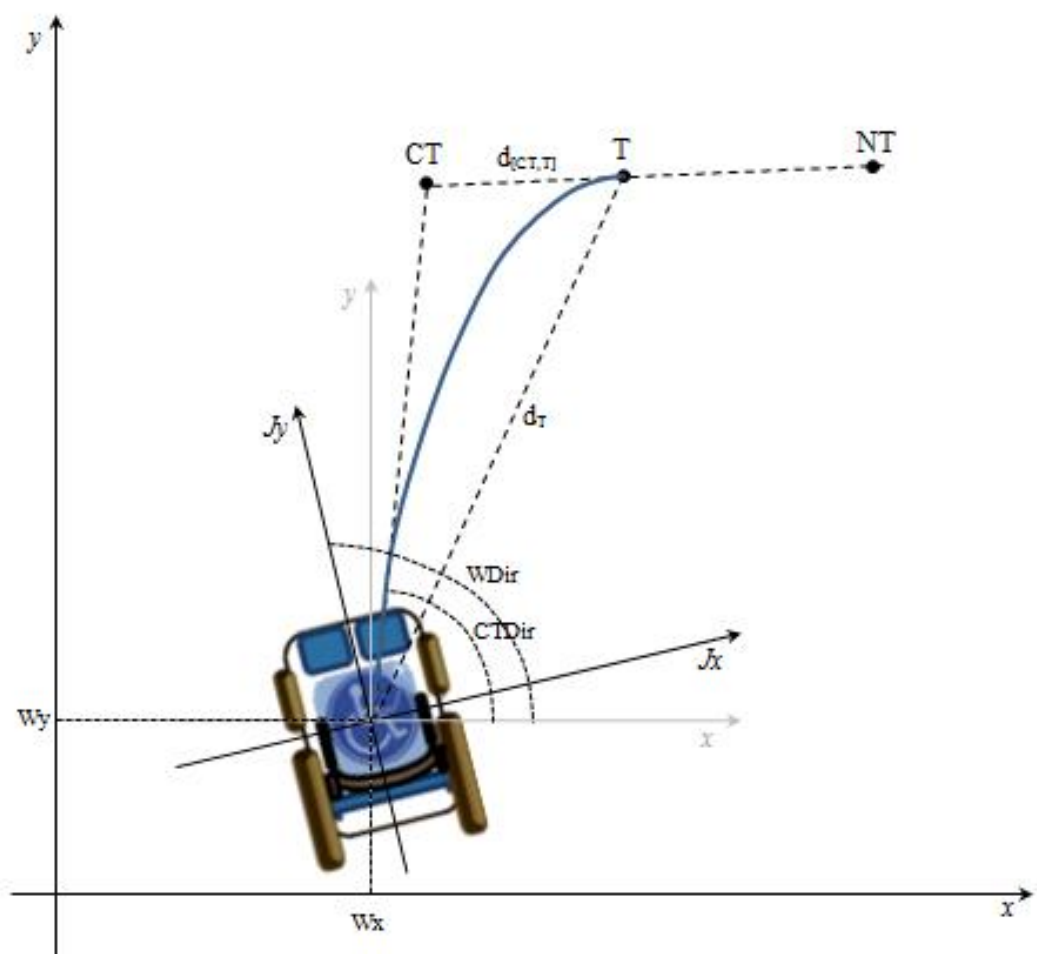

Figure 3. Automatic action of following circuit

The corrected target is calculated using a ramp function as shown in Fig. 3 and has the same direction as the target (T) to the next target (NT).

$$
d_{[C T, T]}=\left\{\begin{array}{l}
0 \quad \text { if } 0<d_{T}<a \\
\frac{d_{\max }}{b-a} \mathrm{~d}_{\mathrm{T}}-\frac{a \times d_{\max }}{b-a} \text { if } a \leq d_{T}<b \\
d_{\max } \text { if } b \leq d_{T}<+\infty
\end{array}\right.
$$


A maximum distance $d_{[C T, T]}\left(d_{\max }\right)$ could be chosen, considering for example the surrounding obstacles. Figure 4 displays the values empirically adjusted and used for the experiments after the first tests with the algorithm.

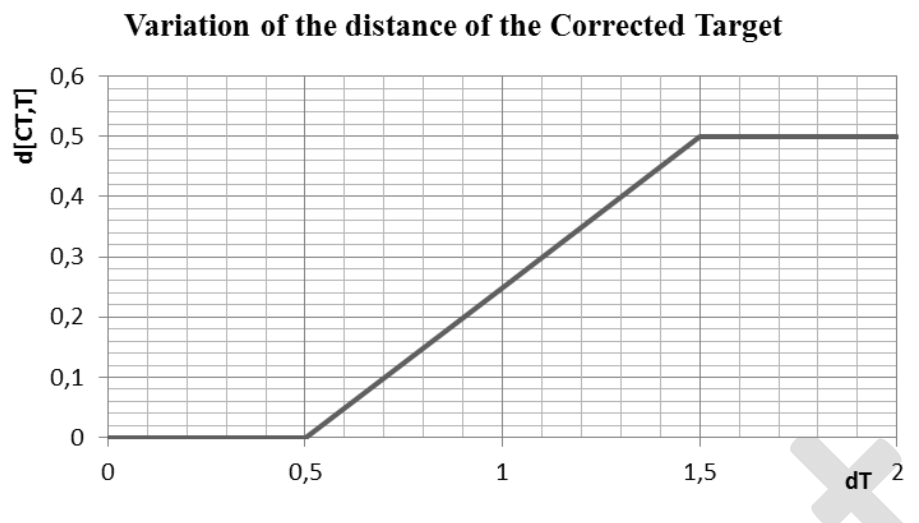

Figure 4. Variation of the distance of the corrected target

In this figure $d_{\max }$ has a value of 0.5 units, $\mathbf{a}$ has the value 0.5 and $\mathbf{b}$ has the value 1.5 .

The distance of the target to the corrected target is determined by the value of the ramp function using the distance of the wheelchair to the target $\left(d_{T}\right)$ as input. Knowing this distance, the position of the corrected target may be determined by projecting the point along the next target to target line at this distance from the target. Using this information and the difference between wheelchair direction (WDir) and the angle to the correct target it is possible to calculate the correct target relative direction (CTDir), and using this value it is possible to calculate the difference between the joystick position direction and the CTDir direction. This difference is afterwards used for the shared control algorithm.

\subsection{Shared Control}

The concept behind the implemented shared control is to understand the intention of the user while providing an easier and safer navigation. This means that, for example, if a user has a high level of difficulty in driving the IW but his intentions can be recognized, the shared control helps the navigation of the wheelchair. Additionally the wheelchair takes control when the navigation of the patient endangers its own safety, in situations such as potential collisions with objects. The computer momentarily takes control and acts on the wheelchair, taking into account the information from sensors and the commands from the user.

A more concrete example is given in Figure 5. If the position of the joystick is at a higher distance than a given threshold from the automatic control command then 
it uses the user command otherwise it uses a weighted average of the automatic control and manual control.

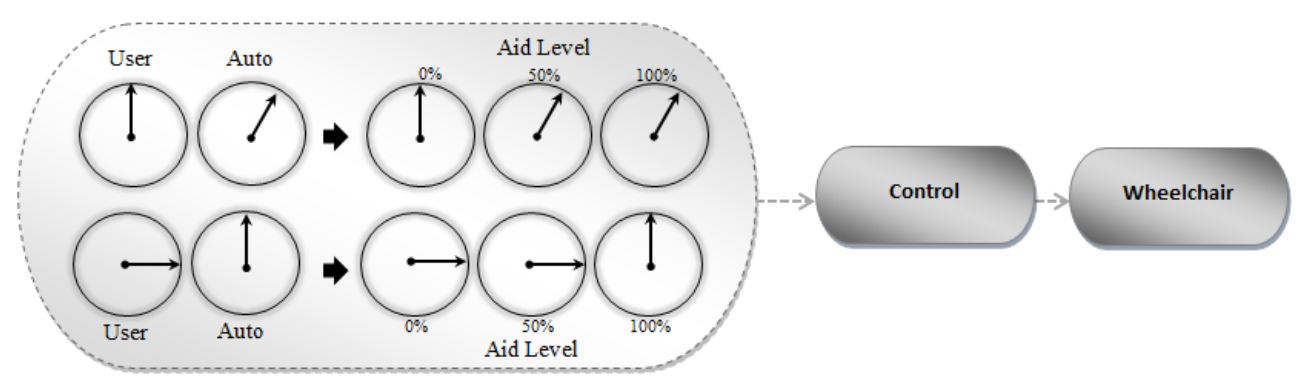

Figure 5. Aided control relatively to the user and to the automatic control

The weights used in the weighted average determine the aid level that is provided to the user. Algorithm 1 presents the shared control implementation.

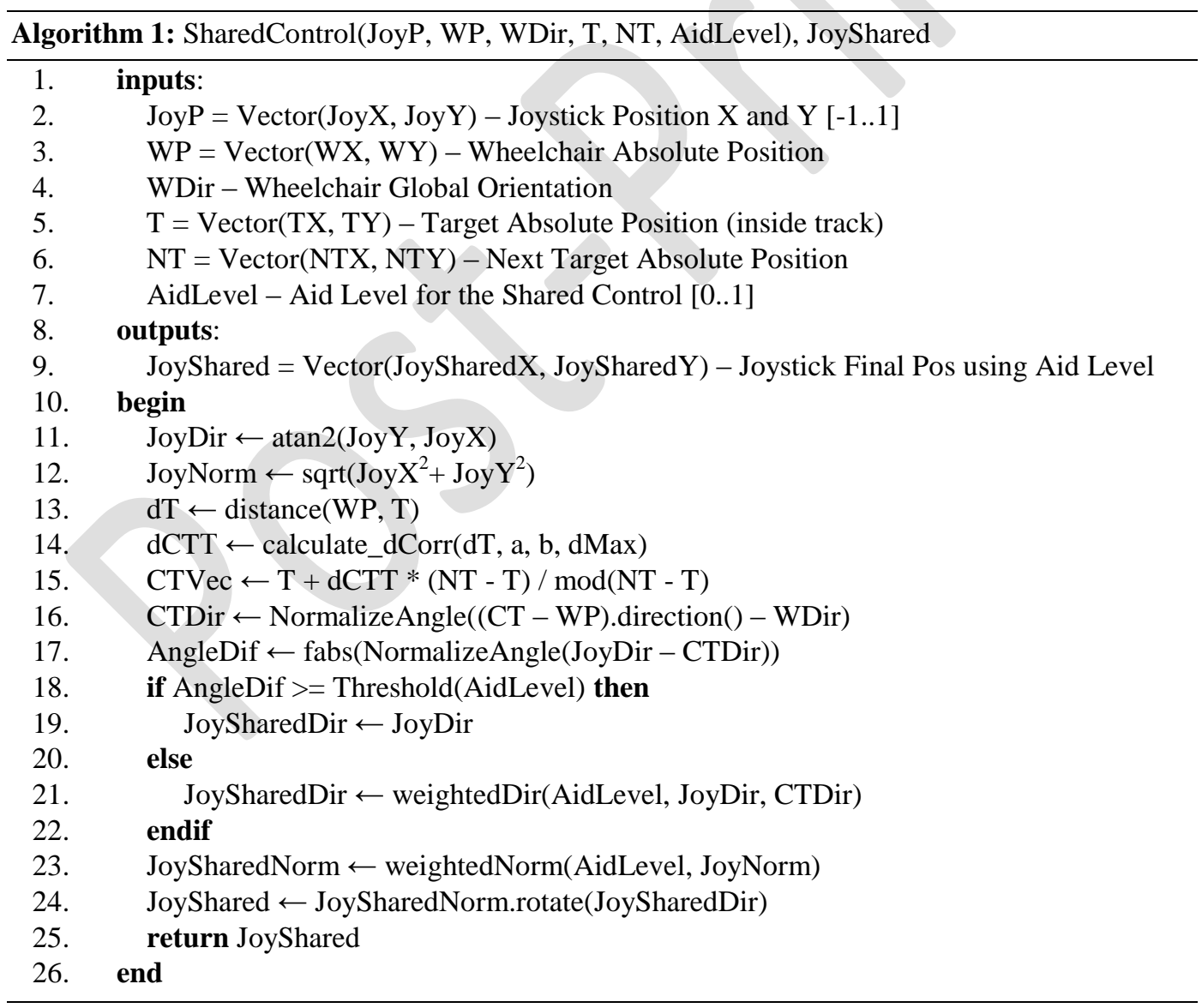

The algorithm receives the joystick position (representing the user intended action), the wheelchair global position (WP) and orientation (WDir), the target and next target points and the aid level considered- It then calculates the Joystick shared control position using this information. 
The shared control algorithm starts by calculation the joystick position direction and norm. Then, it calculates the distance between the wheelchair current position and its target point (dT). Using this distance and the parameter values for $\mathrm{a}, \mathrm{b}$ and dMax, dCTT is calculated and then equation 3 is applied in order to calculate the corrected target.

The angle difference between the wheelchair heading and the corrected target is then calculated and the normalized absolute difference between this angle and the joystick direction is used to estimate the difference between tha automatic control suggestion and the user intention. If the angle difference is above a given threshold that depends on the aid level the user action is used as the final action. Otherwise a weighted direction is calculated based on the aid level, user joystick direction and corrected target direction. For example, for a very high aid level, this function considers that the joystick shared direction will be exactly the corrected target direction, enabling users with imprecise control of the joystick to do very precise driving of the wheelchair. The last step of the algorithm is to weight the joystick shared vector using the user joystick position in order to enable the user to keep some control over the wheelchair velocity even with very high aid levels.

The final joystick corrected position using the shared control is then used as input for the control module that is able to used distinct joystick position to adjust wheels velocities. The implementation of the shared control also considered the obstacle avoidance procedure with the information of the sensors present in the IW. With this tool a more confident way of driving the IW is executed by the users with severe disabilities.

\subsection{Wheelchair Control Advisor}

The idea of having an aided control, for each patient, emerged from the experiments with real people with physical constraints. In fact, there were several patients that had some kind of movement ability, however not precise enough for driving a wheelchair in a safe manner. The aided control was implemented in a simple but effective manner and the adaptation for each user was performed using a profile module [23]. The profile module is available in the multimodal interface (Figure 6) and using it several simple tasks test the patient ability in moving the 
joystick and performing head movements in several directions such as "North", "South", "East" and "West".
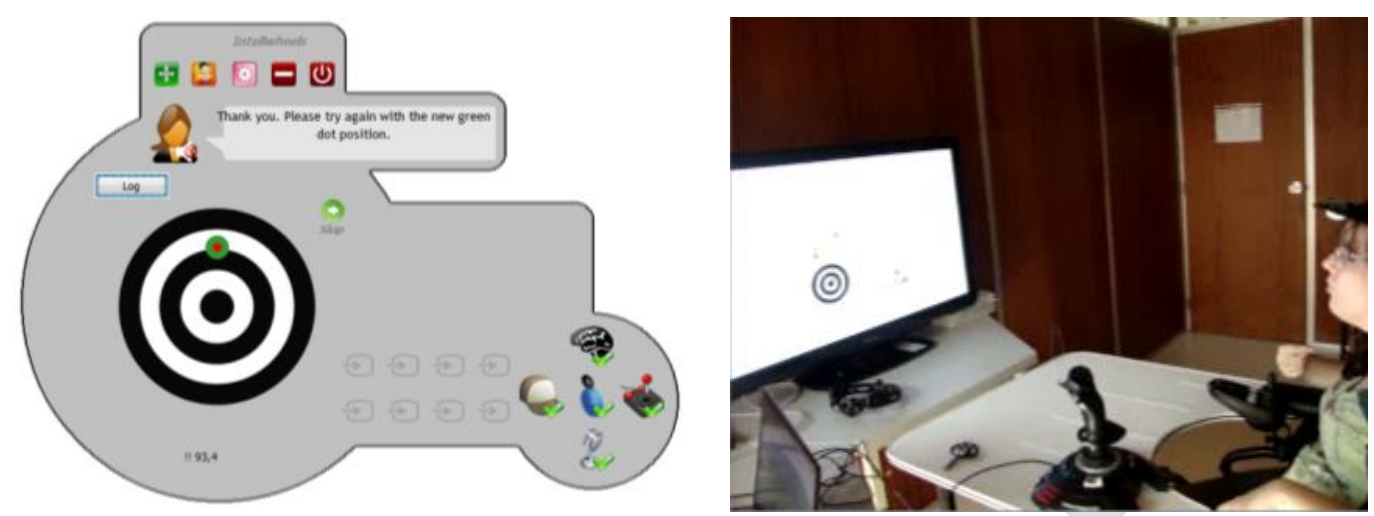

Figure 6. Profile module incorporated in the multimodal interface

The wheelchair control advisor provides several alternatives considering the different accuracy levels achieved by the specific user:

- Shared control with an aid level of $100 \%$ (with obstacle avoidance) - if the overall accuracy is included in $[0, x[$;

- Shared control with an aid level of 50\% (with obstacle avoidance) - if the overall accuracy is included in $[x, y[$ where $0<x<y<1$;

- Manual control (with obstacle avoidance) - if the overall accuracy is in the interval $[y, 1[$.

Obstacle avoidance is always advised using shared control, since it increases the safety of the user. In particular, if the accuracy has the value between $30 \%(x)$ and $70 \%(y)$ then the aided control advised is at a level 50\%. If the user selects an input device with very low accuracy, and that was not one of the advised input devices, then the wheelchair control informs that the best choice is to use the aided control at a $100 \%$ level. These parameters were obtained using supervised classification helped by the occupational therapists.

\section{Experiments and Results}

The experiments using the simulator IntellSim and the profile module allowed testing the users' ability in driving the wheelchair with several input devices. After that it was possible to verify if an extra help should be provided to the user. It is possible to provide the users with the shared control with distinct aided levels as explained in Section 3.4. The automatic control could be used, for example, by 
the patient's escort such as a relative or medical personal in order to avoid them from the need to manually push the wheelchair to the intended target.

The experiments with the shared control aimed at testing how the users with cerebral palsy would react when having a control that helps them in the task of driving wheelchair. The usability level was determined based on users' feedback after testing the different shared controls without previously knowing the control characteristics: aided control at a $100 \%$, aided control at a 50\% and manual with obstacle avoidance. The experiments were conducted using the IntellSim and the order of tests with the shared controls was set randomly. A circuit was defined and several objects to be collected (passing near them) were put along the way. These objects (27 blue balls and a star in Figure 7) determine the path that the wheelchair should follow.

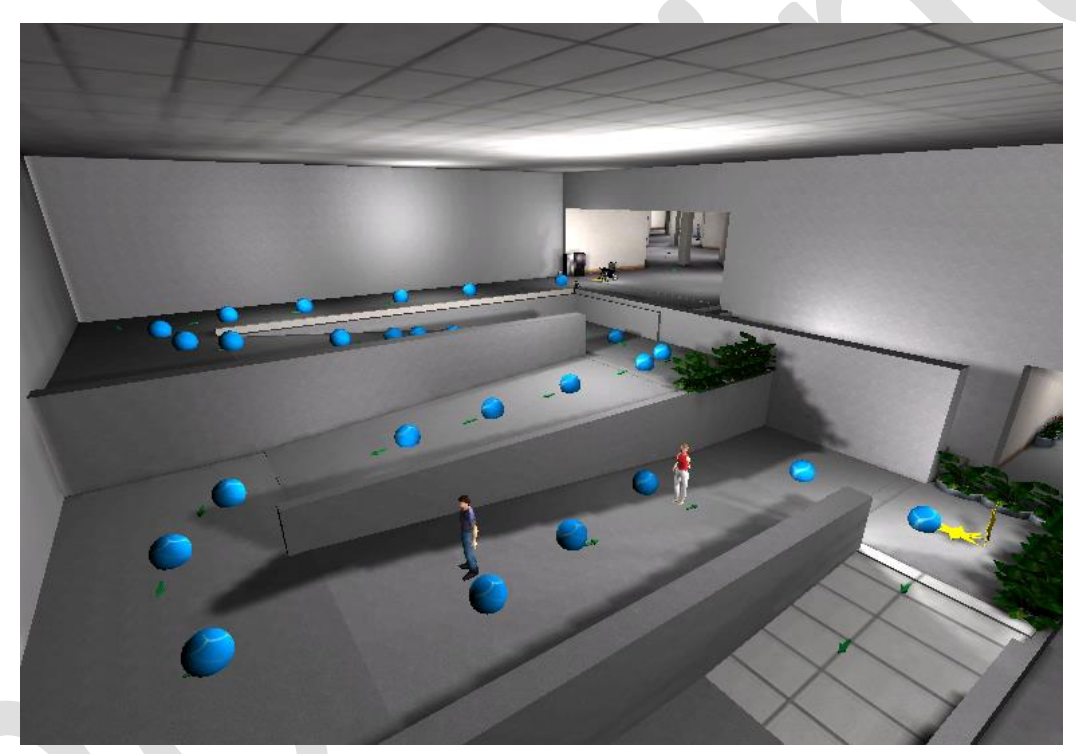

Figure 7. Circuit for testing the shared controls

The patients tested the shared controls using the joystick as input device and using head movements detected by a wiimote (Figure 8).
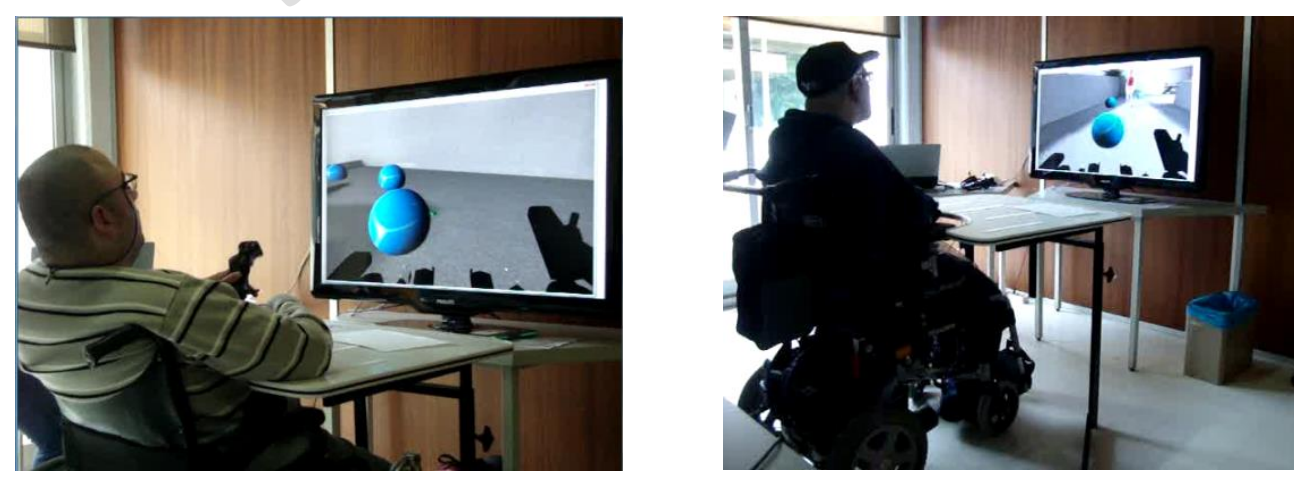

Figure 8. Simulator users' trials 
After each round the patients answered a questionnaire composed of four parts: user identification; experience with videogames and joysticks; questions adapted from the Computer System Usability Questionnaire (CSUQ) [27] for each tested option and a final question about the preference order of the tested options.

The sample was a group of 8 cerebral palsy patients with the level IV and V of the Gross Motor Function Measure [28]. This sample is characterized by having six males and two females, with a mean of age of 31 years old. All had experience with the joystick of their electric wheelchair although the experience with video games was low, except in one case that answered always play videogames.

Table 2 shows the summary of statistics measures about the final score for all the mapping options. The obtained results from the final score of the CSUQ show a tendency to consider the aided control at a $50 \%$ the best way to drive the wheelchair with the joystick and the wiimote. In general, the opinions considered all options very useful except in the case of the head movements with obstacle avoidance which had the worst result. Nevertheless it is possible to affirm that the cerebral palsy patients would react favorably when having a control that helps the driving of the wheelchair.

Table 2. Summary of statistical measures of the adapted CSUQ score

\begin{tabular}{|c|c|c|c|c|c|c|}
\hline \multicolumn{7}{|c|}{ Adapted CSUQ - Final Score } \\
\hline Statistics & $\begin{array}{c}\text { Aid Cont. } \\
100 \%\end{array}$ & $\begin{array}{c}\text { Aid Cont. } \\
50 \%\end{array}$ & $\begin{array}{c}\text { Obs. } \\
\text { Avoid. }\end{array}$ & $\begin{array}{c}\text { Aid Cont. } \\
100 \%\end{array}$ & $\begin{array}{c}\text { Aid Cont. } \\
50 \%\end{array}$ & $\begin{array}{c}\text { Obs. } \\
\text { Avoid. }\end{array}$ \\
\hline Mean & 82.7 & 87.1 & 84.1 & 76.0 & 76.4 & 50.6 \\
\hline Median & 88.9 & 99.2 & 88.9 & 84.9 & 91.3 & 41.3 \\
\hline Std. Dev. & 21.0 & 22.3 & 19.4 & 24.7 & 33.1 & 30.8 \\
\hline Min & 39.68 & 36.5 & 44.4 & 31.8 & 15.9 & 14.3 \\
\hline Max & 100 & 100 & 100 & 100 & 100 & 96.83 \\
\hline
\end{tabular}

In order to confirm the differences between the shared controls using joystick and wiimote, the Friedman test (related samples Friedman's test two way analysis of variance by ranks) was applied to the final scores. The $\mathrm{p}$ values were 0.484 and 0.004, and for that reason there are no statistical evidences to affirm that the distributions of the scores are significantly different for the joystick shared controls and there are statistical evidences to affirm that the distributions of the scores are significantly different for the head movements shared control at a level of 0.05 .

Table 3 shows the $p$ values of the multiple comparisons, using the Fisher's least significant difference (LSD) in the case of the head movements shared controls. 
Table 3. Multiple comparisons of the head movements shared controls

\begin{tabular}{|c|c|c|}
\hline \multicolumn{3}{|c|}{ Multiple Comparisons LSD Head movements shared controls(p values) } \\
\hline & Aid. Control $100 \%$ & Aid. Control 50\% \\
\hline Aided control $50 \%$ & 1 & -- \\
\hline Obstacle Avoidance & 0.001 & 0.001 \\
\hline
\end{tabular}

The results of the CSUQ score also confirm the tendency to the order of preference as can be observed in Table 4.

Table 4. Summary statistics about the order of preference of the shared controls

\begin{tabular}{|c|c|c|c|c|c|c|}
\hline \multicolumn{7}{|c|}{ Adapted CSUQ - Final Score } \\
\hline \multirow[b]{2}{*}{ Statistics } & \multicolumn{3}{|c|}{ Joystick } & \multirow[b]{2}{*}{$\begin{array}{c}\text { Aid Cont. } \\
100 \%\end{array}$} & \multicolumn{2}{|c|}{ Wiimote } \\
\hline & $\begin{array}{c}\text { Aid Cont. } \\
100 \%\end{array}$ & $\begin{array}{c}\text { Aid Cont. } \\
50 \%\end{array}$ & $\begin{array}{c}\text { Obs. } \\
\text { Avoid. }\end{array}$ & & $\begin{array}{c}\text { Aid Cont. } \\
50 \%\end{array}$ & $\begin{array}{c}\text { Obs. } \\
\text { Avoid. }\end{array}$ \\
\hline Median & 2 & 2 & 3 & 1 & 2 & 3 \\
\hline Min & 1 & 1 & 1 & 1 & 2 & 1 \\
\hline Max & 2 & 3 & 3 & 2 & 3 & 3 \\
\hline
\end{tabular}

The aided control was chosen as the best way of driving the wheelchair in the case of using the joystick and the wiimote for the head movements. It was interesting to verify that all the patients found the experience of the aided control very pleasant.

In terms of performance, all objects were collected using the joystick in the three controls modalities. Using the head movements, all the objects were collected using the aided controls at $100 \%$ and $50 \%$, against a mean value of 25.75 objects (a minimum of 18 objects and the maximum of 28 objects) when using the manual control with obstacle avoidance.

Table 5 shows three users' circuits tracking performed using joystick and head movements with the three available shared controls.

Table 5. Circuits tracking using joystick and head movements with three shared controls

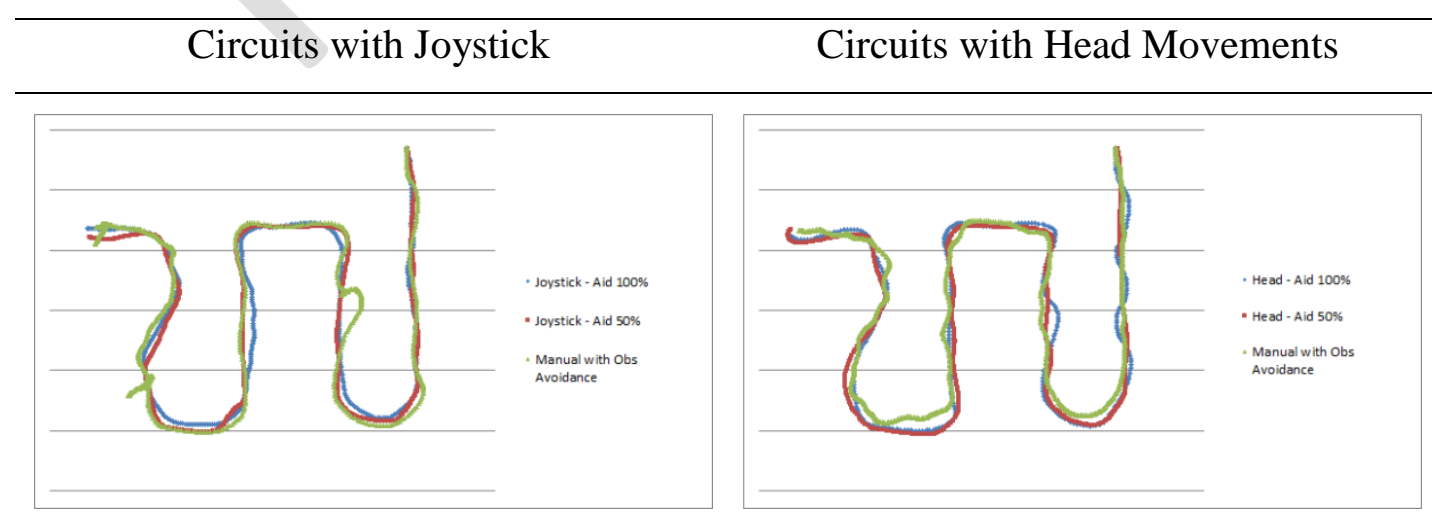




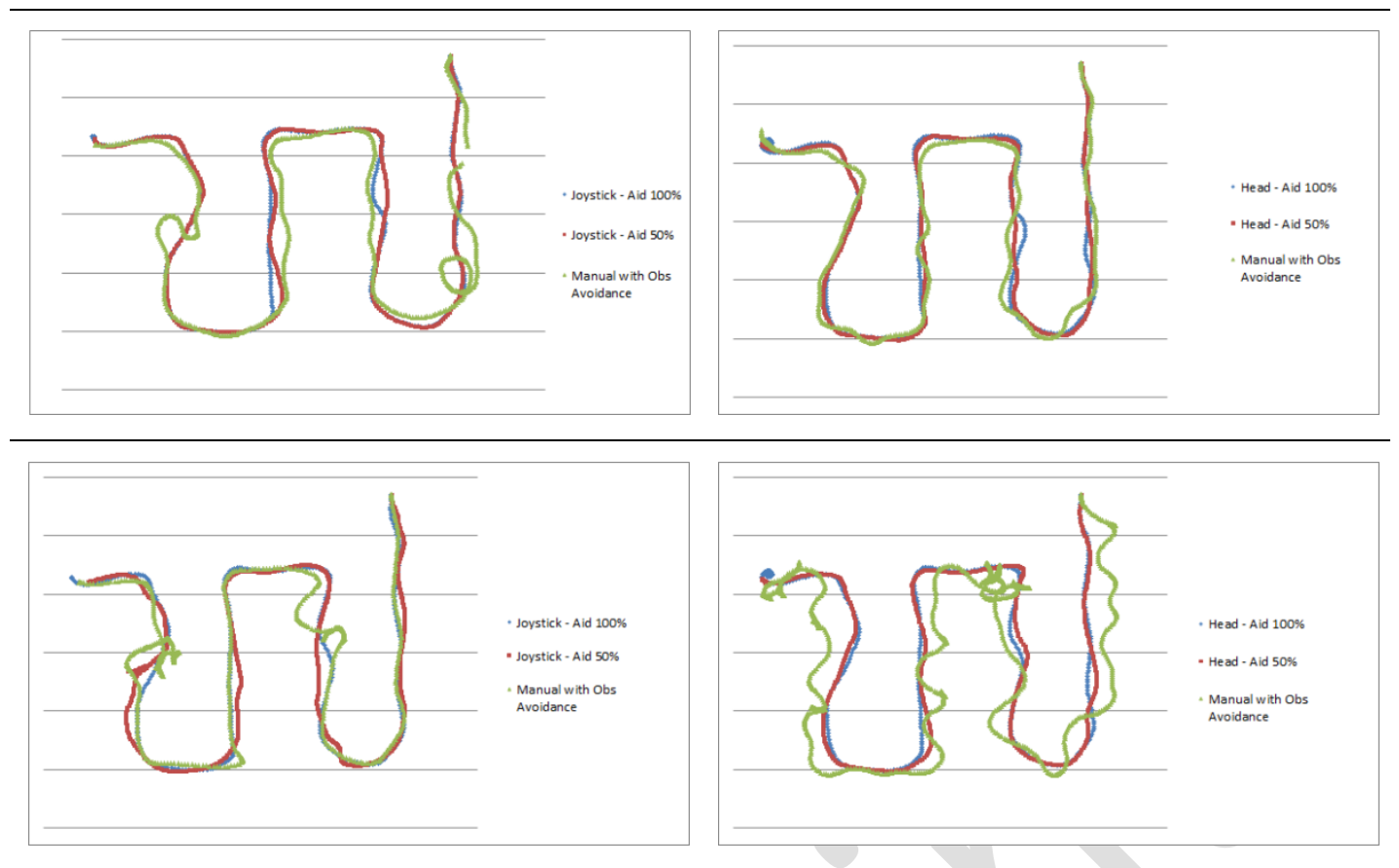

Observing the circuits it is clear the higher error in following the trajectory using the manual control with obstacle avoidance. Although the manual control with obstacle avoidance and using head movements had the worst preference and usability, there are users, such as the second user in Table 5, that have a behavior more accurate using head movements than using the joystick movements. This also revealed the importance of giving different choices and a multimodal interface for driving the intelligent wheelchair.

A careful observation of the experiments executed by the patients was also performed by the occupational therapists and some interesting notes are important to register. All the users think that they had control of the wheelchair even when they were using the aided control at a level of $100 \%$, except one case in which the user had obvious involuntary movements. He found it very strange that the wheelchair had such as smooth behavior and stopped a few times to check if the wheelchair corresponded to his action. Another situation was a case of a patient that had cognitive deficits; the level of motivation was very high when using the shared control at a level $100 \%$ and this could be identified by his non-verbal language.

\section{Conclusions and Future Work}

The shared control experiments revealed the importance of using aided controls for users with severe disabilities. The patients still felt having complete control over the wheelchair movement when using a shared control at a 50\% level. Thus, 
this control type was very well accepted and may be used as the main control method for the IW prototype. Overall the responses were very positive to this kind of control in terms of usability and preference. It was concluded that it is preferable a more intuitive reaction of the wheelchair to the position of the joystick and head positions. Moreover, the shared control should be used to have a higher safety when a user with physical limitations is driving the intelligent wheelchair. The shared control with adequate aid level for driving the wheelchair can be a usable solution to help the daily life chores of users.

The input control advisor can also help in the initial decision to select an interface and the aided control level for handicapped people. This can help the user and the occupational therapist that normally follows the wheelchairs' users.

In this study, the shared control was only used in the simulated environment. However, the future evolution of the project will enable to have the localization issues of the real wheelchair solved in order to allow the aided control also in the real environment.

Future work will be concerned with conducting a deeper study of the control methods by testing different configuration parameters for each control type and by testing the control methods with a broader sample of wheelchair users. Another experiment will be concerned with the use of machine learning algorithms to create user driving models and using them to create automatic control methods based on real user behavior. Finally it will be interesting to test the behavior with more complex paths and without any predefined paths. Also it may be interesting to conduct experiments using only the final target and how the system can help the user, on this situation, with different shared controls.

Acknowledgments: This work was funded by the ERDF - European Regional Development Fund through the COMPETE Programme (operational programme for competitiveness) and by National Funds through FCT - Portuguese Foundation for Science and Technology within project «INTELLWHEELS - Intelligent Wheelchair with Flexible Multimodal Interface, RIPD/ADA/109636/2009». B. M. Faria thanks to FCT for the PhD Scholarship FCT/SFRH/BD/44541/2008

\section{References}

[1] Faria, B.M., Ferreira, L., Reis, L.P., Lau, N., Petry, M. and Soares, J.C.: Manual Control for Driving an Intelligent Wheelchair: A Comparative Study of Joystick Mapping Methods, IROS 2012, Progress, challenges and future perspectives in navigation and manipulation assistance for 
robotic wheelchairs workshop, IEEE/RSJ International Conference on Intelligent Robots and Systems 2012, Vilamoura, Algarve, pp. 7-12 (2012).

[2] Petry, M., Moreira, A. P., Braga, R. and Reis, L. P.: Shared control for obstacle avoidance in intelligent wheelchairs, in IEEE Conference on Robotics, Automation and Mechatronics, Singapore, pp. 182-187 (2010).

[3] Simpson, R.: Smart wheelchairs: A literature review, Journal of Rehabilitation Research \& Development, vol. 42, no. 5, pp. 423-435 (2005).

[4] Woods B. and Watson N.: The social and technological history of wheelchairs, International Journal of Therapy and Rehabilitation, vol. 11, no. 9, pp. 407-410 (2004).

[5] Faria, B.M., Vasconcelos, S., Reis, L.P. and Lau, N.: Evaluation of Distinct Input Methods of an Intelligent Wheelchair in Simulated and Real Environments: A Performance and Usability Study. Assistive Technology: The Official Journal of RESNA (Rehabilitation Engineering and Assistive Technology Society of North America), USA, vol. 25, no. 2, pp. 88-98 (2013)

[6] Simpson, R., LoPresti, E., Hayashi, S., Nourbakhsh, I. and Miller, D.: The Smart Wheelchair Component System, Journal of Rehabilitation Research \& Development, vol. 41, no. 3B, May/June, pp. 429-442, (2004).

[7] Braga, R., Petry, M., Moreira, A. P. and Reis, L. P.: Concept and Design of the Intellwheels Platform for Developing Intelligent Wheelchairs, in LNEE/ Informatics in Control, Automation and Robotics, vol. 37, pp. 191-203, (2009).

[8] Madarasz, R.L., Heiny, L.C., Cromp, R.F. and Mazur, N.M.: The design of an autonomous vehicle for the disabled, IEEE Journal of Robotics and Automation, vol. 2, no. 3, September, pp. 117-126 (1986).

[9] Hoyer, H. and Hölper, R.: Open control architecture for an intelligent omnidirectional wheelchair,” in Proc.1st TIDE Congress, Brussels, pp. 93-97, (1993).

[10] Wellman, P., Krovi, V. and Kumar, V.: An adaptive mobility system for the disabled, in Proc. IEEE Int. Conf. on Robotics and Automation, vol 3, pp. 2006-2011, (1994).

[11] Simpson, R.: NavChair: An Assistive Wheelchair Navigation System with Automatic Adaptation, in Assistive Technology and Artificial Intelligence, Berlin, Springer-Verlag Berlin Heidelberg, p. 235 (1998).

[12] Miller, D. and Slack, M.: Design and testing of a low-cost robotic wheelchair, in Autonomous Robots, vol. 2, no. 1, pp. 77-88, (1995).

[13] Miller, D.: Assistive Robotics: An Overview, in Assistive Technology and AI, vol. 1458, pp. 126-136, (1998).

[14] Borgerding, B., Ivlev, O., Martens, C., Ruchel, N. and Gräser, A.: FRIEND: Functional robot arm with user friendly interface for disabled people, in 5th European Conf. for the Advancement of Assistive Technology (1999).

[15] L. Project: LURCH - the autonomous wheelchair, [Online]. Available:http://airwiki.ws.dei.polimi.it/index.php/LURCH_The_autonomous_wheelchair.

[Accessed May 2013].

[16] Bonail, B., Abascal, J. and Gardeazabal, L.: Wheelchair-based open robotic platform and its performance within the ambiennet project, in Proceedings 2nd International Conference on 
Pervasive Technologies Related to Assistive Environments, ACM: Corfu, Greece, pp. 631-636 (2009).

[17] Pruski, A., Ennaji, M. and Morere, Y.: VAHM: A user adapted intelligent wheelchair, in Proceedings of the 2002 IEEE International Conference on Control Applications, Glasgow, vol. 2, pp. 784-789, (2002).

[18] Soh, H. and Demiris, Y.: Towards Early Mobility Independence: An Intelligent Paediatric Wheelchair with Case Studies, in IROS 2012 Workshop on Progress, Challenges and Future Perspectives in Navigation and Manipulation Assistance for Robotic Wheelchairs, Vilamoura, (2012).

[19] Mandel, C., Rofer, T. and Lohmuller, I.: On the Clinical Evaluation of Smart Driving Assistance for Power Wheelchairs, in IROS 2012 Workshop on Progress, Challenges and Future Perspectives in Navigation and Manipulation Assistance for Robotic Wheelchairs, Vilamoura, (2012).

[20] Braga, R., Petry, M., Reis, L. P., Moreira, A. P.: IntellWheels: Modular development platform for intelligent wheelchairs, Journal of Rehabilitation Research \& Development, vol. 48, no. 9, pp. 1061-1076 (2011).

[21] Braga, R., Petry, M., Moreira, A. P., Reis, L. P.: Intellwheels: A Development Platform for Intelligent Wheelchairs for Disabled People. Proceeding of the 5th International Conference on Informatics in Control, Automation and Robotics. Vol I. Funchal, Madeira, Portugal, pp.115-121 (2008).

[22] Petry, M., Moreira, A. P., Reis, L. P., Rossetti, R.: Intelligent Wheelchair Simulation: Requirements and Architectural Issues, in 11th International Conference on Mobile Robotics and Competitions, Lisbon, pp. 102-107, (2011).

[23] Faria, B. M., Vasconcelos, S., Reis, L. P., Lau, N.: A Methodology for Creating Intelligent Wheelchair Users' Profiles, ICAART 2012 - 4th International Conference and Artificial Intelligence, Algarve, pp 171-179 (2012).

[24] Faria, B. M., Reis, L. P., Lau, N., Soares, J. C., Vasconcelos, S., Patient Classification and Automatic Configuration of an Intelligent Wheelchair, Communications in Computer and Information Science 358, Springer-Verlag, pp. 268-282, 2013

[25] Faria, B. M., Reis, L. P., Lau, N.: Knowledge Discovery and Multimodal Inputs for Driving an Intelligent Wheelchair, International Journal of Knowledge Discovery in Bioinformatics, vol. 2, no. 4, pp 18-34 (2011).

[26] Faria, B. M., Reis, L. P., Lau, N.: Manual, Automatic and Shared Methods for Controlling an Intelligent Wheelchair: Adaptation to Cerebral Palsy Users, 13th International Conference on Autonomous Robot Systems and Competitions, pp. 26-31 (2013).

[27] Lewis, J. R.: IBM Computer Usability Satisfaction Questionnaires: Psychometric Evaluation and Instructions for Use, International Journal of Human-Computer Interaction, vol. 7, no. 1, pp. 57-78 (1995).

[28] Palisano, R.J., Rosenbaum, P., Bartlett, D., and Livingston, M.H.: Content validity of the expanded and revised Gross Motor Function Classification System, Development Medicine and Child Neurology, vol. 50, no. 10, pp. 744-750 (2008). 